\title{
The Reflexive Campus: A Study of Dynamic Architecture in a Virtual World
}

\author{
Elif Ayiter \\ Sabanci University \\ ayiter@sabanciuniv.edu
}

\author{
Selim Balcisoy \\ Sabanci University \\ balcisoy@sabanciuniv.edu
}

\author{
Murat Germen \\ Sabanci University \\ muratgermen@sabanciuniv.edu
}

\author{
Bulut Sakcak \\ Sabanci University \\ buluts@su.sabanciuniv.edu
}

\author{
Fethi Ozdol \\ Sabanci University \\ fethiozdol@su.sabanciuniv.edu
}

\begin{abstract}
This paper describes the creative as well as computational processes involved in the creation of a generative architectural construct destined to become the virtual campus of a Real Life University in Second

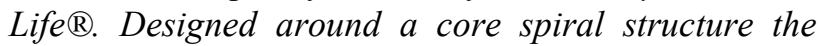
virtual building re-configures itself based upon user demand, adding exhibition and meeting areas as well as conference halls and auditoriums, as and when required. Thus, a user-centric, emergent, dynamic construct has been implemented for a virtual campus, which evolves and changes over time and usage. The ensuing design system also attempts to configure itself based upon the nature of activity undertaken within it: Thus spaces required for survey based learning spread themselves out over the "shallow" horizontal axis, whereas areas of research and deeper levels of inquiry utilize the "deeper" vertical axis in a visual representation of different strategies of learning and levels of epistemological inquiry.
\end{abstract}

\section{Introduction}

The Reflexive Campus has been designed as part of the course work of an undergraduate university course, bringing together students majoring in Fine Arts/Design and Computer Science, to form teams of two, undertaking the development of projects which integrate expertise from both these fields, culminating in novel areas of creative endeavor. Since the learning strategies of this course have been discussed elsewhere $[01$, beyond noting that it is this unique hybrid learning environment which brought together a computer scientist and an artist in the creation of this project, they will not be discussed further here.

\subsection{Formal Investigations: Morphogenetic attributes of the Spiral}
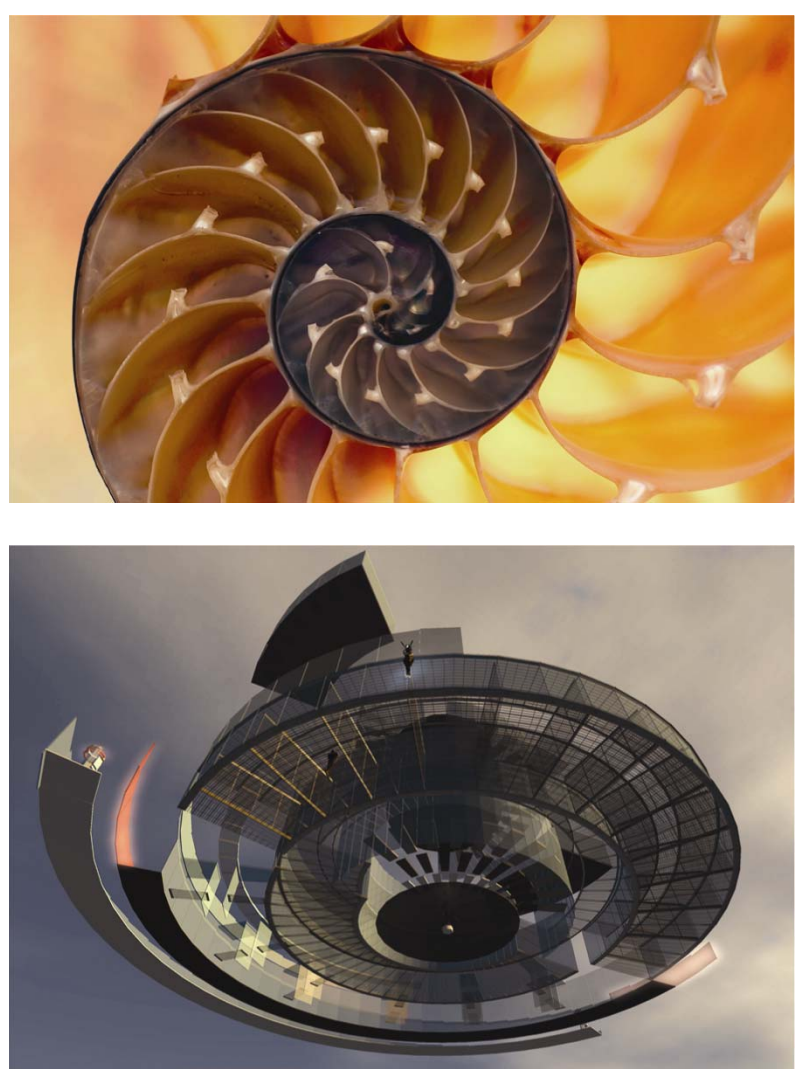

Figure 1: The spiral as a natural form of growth (above) and the spiral as the primary generative module of the Campus (below). 


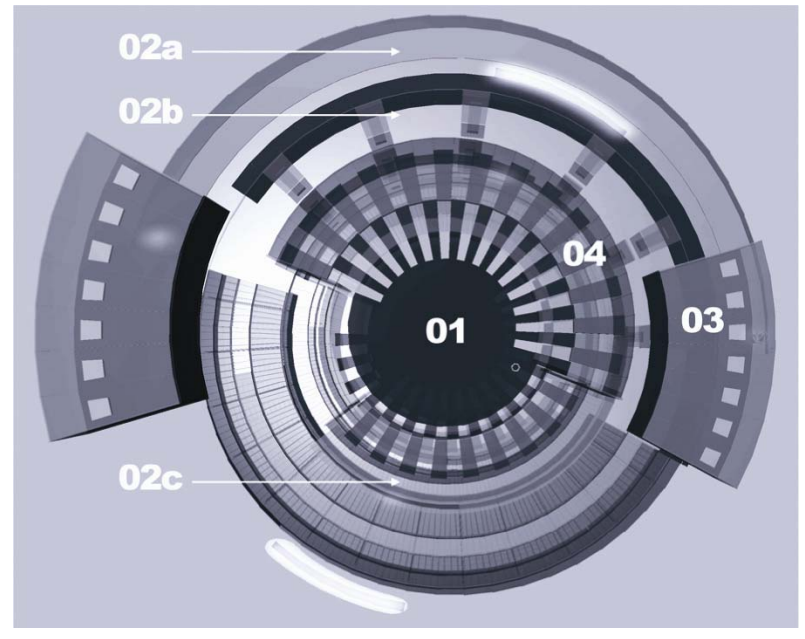

Figure 2: Reflexive Campus, schematic diagram: 01 Central Atrium, $02(a, b, c)$ The three rotational rings forming a spiral, 03 Small classroom rendered on the horizontal axis in a sunray system from the pivot, 04 Large Auditorium rendered on the vertical axis, aligned to pivot.

Inspired by two buildings, one real - the Spiral Minaret of the Great Mosque at Samarra, built in 847 $\mathrm{AD}$, and one imaginary - the Tower of Babel as envisioned by Brueghel the Elder, in his so called painting from 1563; a spiral was decided upon as the primary building module around which the entire construct would be generated

However, it was not solely the fascination with these buildings that further prompted this decision: When formal investigations commenced it was found that the continuity inherent in the circular and yet ever expanding shape of the spiral was uniquely suited to the demands of the task at hand, which was one of creating a construct capable of infinitely enlarging itself, capable of rendering parts of itself over and over again, and yet capable of maintaining its holistic structure throughout the generative process. An examination of Morphogenesis and Architecture provided ample validation for the implementation of a spiral as the primary form of architectural growth, from which the campus would flourish, since the spiral is a shape also found in patterns of natural growth. [02].

\subsection{The Liquid Architecture of Cyberspace}

Validation for the pursuit of a dynamic architecture in the virtual realm can be found in the proclamations of Toyo Ito:
... Architecture in the Electronic Age is the Figuration of a Vortex of Information

... Architecture in the Electronic Age Changes the Concept of Barrier

... Architecture in the Electronic Age is Architecture That Designs Time

... Architecture has traditionally been linked with nature through figuration of movements of vortices occurring in water and air. With contemporary architecture, we must link ourselves with the electronic environment through figuration of information vortices...

... For, just as the figure of a living body represents the loci of movements of air and water, virtual spaces will most likely be figured as the loci of human activities in the electron flow.

Toyo Ito, 1997, from "Image of Architecture in the Electronic Age" [03]

Theoretician/architect Marcus Novak, harkens back to El Lissitsky: "Proun is a transfer station from painting to architecture, the 'immaterial material". This immaterial material, born from light and the motion of objects, has elements in common with the space of electronic media. "The liquid architecture of cyber space is clearly immaterial architecture. It is architecture that is no longer satisfied with form, light and the other aspects of the real world. It is an architecture composed of changing relationships between a variety of abstract elements" [04].

Finally, in terms of educational spaces, validation of the pursuit of experimental forms that may aid experiential learning can be found in John Dewey's learning theories [05]. Dewey puts high value on the structuring of the physical educational as well as social environment and its operant components, proclaiming that "in last analysis, all that the educator can do is modify stimuli so that response will as surely as is possible result in the formation of desirable intellectual and emotional dispositions", creating environments where experience may be fruitfully rewarded and kept continuously active, as well as its outcome closely monitored. Thus structures within which these activities can be accomplished need to be provided and these call for the formulation of a sturdy visual language that will engender a cohesive gestalt, as the design process unfolds,

\subsection{Second Life ${ }^{\circledR}$ and the State of the Art of Reflexive Architecture in the Metaverse}

Reflexive Architecture, according to Neil Spiller, is architecture which is highly responsive and intelligent, able to translate and connect to its contextual and 
environmental surroundings at a new level, while also operating in three or more spaces simultaneously. It is effectively an architecture that is wholly visionary rather than being grounded by the limitations of the present. [06].

While the bulk of Second Life building does not seem to be taking advantage of the affordances of being situated in a virtual, coded environment; remaining resolutely static, there are nonetheless notable exceptions: Just as considerable research in Reflexive Architecture is being conducted in the physical realm, in Second Life the practice of Reflexive Architecture to date has been particularly embodied through the work of architect Jon Brouchoud, aka. Keystone Bouchard, who writes that in physical reality, architecture is a static and relatively motionless artifact. The occupant plays a passive role, observing but rarely impacting its composition, whereas in a virtual environment, the architecture is capable of transcending the limitations of static buildings, and become as fluid and dynamic as the communities of people they hope to serve. Reflexive Architecture is only one of many branches of opportunity for a new language of virtual architecture to emerge, free from the habit of pure physical replication [07].

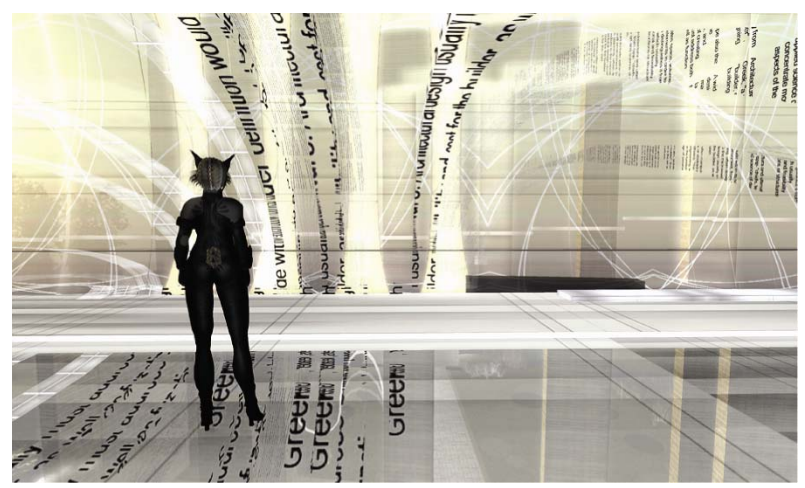

Figure 3: The Reflexive Architecture of Jon Brouchoud.

Yet, while being a strong advocate as well as practitioner of Reflexive Architecture Brouchoud nonetheless alerts us that in an environment where avatars are free to create anything they can imagine, the vast majority of the architectural fabric created is still largely driven by very literal parallels to the physical world. This happens for good reason, as we have learned to visually organize the world around us, real or virtual, based on familiar cues and patterns. A roof may not need to protect us from the elements in virtual space, but it organizes a space. Even though you can fly, a ramp is still a strong way finding mechanism.
While these visual cues from physical reality are imported with good cause, the native, inherent characteristics of this environment and build a new language of virtual architecture based on those characteristics should also be considered. In examining the differences between real and virtual environments, it becomes clear that physical architecture is relatively static and unchanging, whereas virtual architecture has the capacity to become far more dynamic; behaving more like a liquid than a static and passive artifact. "This seemingly simple distinction could serve as a core ingredient and driving force behind a new language of virtual architecture. In this way, the idea of reflexive architecture is not a new language of virtual design, but only a simple diagram that exists within a much larger community-wide exploration of dynamic new forms of virtual architecture".

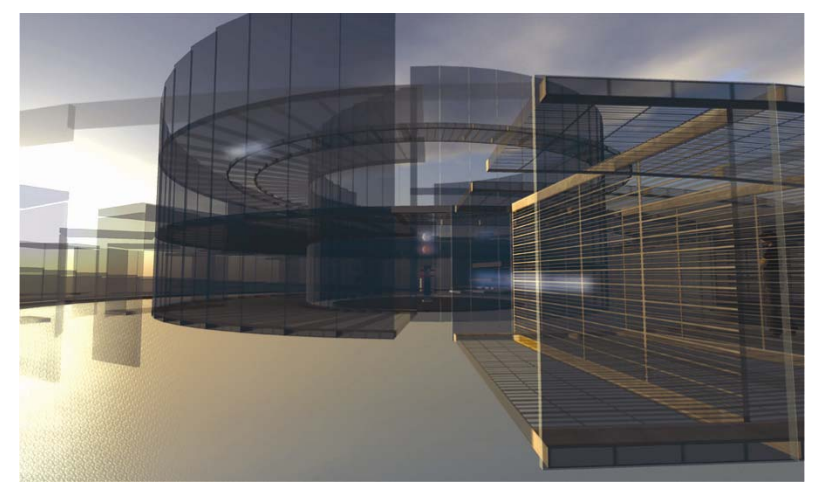

Figure 4: Simulating physical architectural elements such as floors and walkways for orientation and way finding.

Brouchoud's considerations have been taken very much to heart during the design phases of the Reflexive Campus: While the visual language of the construct takes full advantage of all the affordances of the virtual; such as the ability to suspend building components into air and the usage of as locomotion as a structural element, nonetheless a concerted effort at maintaining clearly recognizable physical clues, such as walkways, floors and ceilings, and moreover a central atrium with hallways and passages leading into this center has been made.

\section{The Creative Process}

Challenging Goethe's statement that "architecture is frozen music", the creators of the Reflexive Campus built a construct around a core atrium, of three half rings slowly rotating around this pivotal space, forming a spiral of proportional radiuses, calculated by their 
distance from the pivot. Each of these rings fulfills a separate function: Orienting the visitor, creating a catalyst for interaction and providing exhibition spaces for the university's activities.

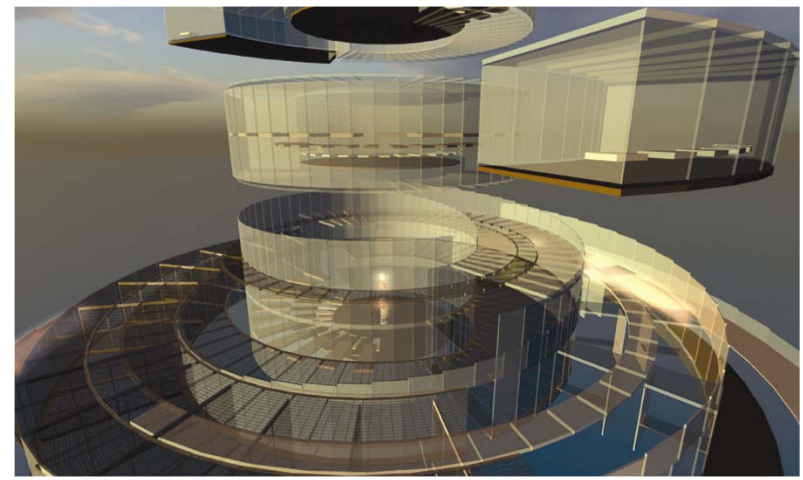

Figure 5: View from an elevated point, with the primary spiral module visible at ground level, with auditoriums and meeting areas suspended in the virtual sky above.

The complex emphasizes the tenets of dynamic architecture by expanding/shrinking, where the size is proportionate to the current occupant population and the shape corresponds to the usage that this population is demanding of the space at that time. Thus, while a day of intensive teaching of undergraduate courses will see a campus spread out over a wide horizontal axis accomplished through the rendering of dedicated modules/classrooms for each of the individual course taught; a day of intensive research activity or conferences will see a campus rising along a vertical axis through the rendering of dedicated auditoriums and meeting areas and seminar rooms. Finally, days of mixed activity and maximum usage will see a campus spreading on both axes.

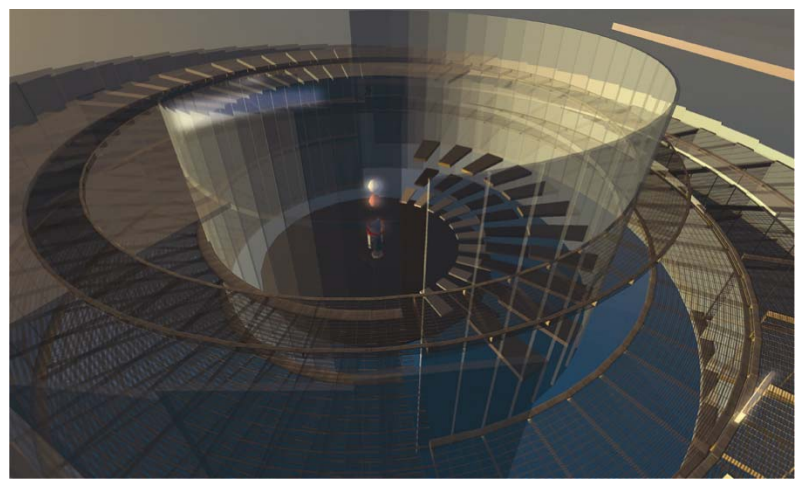

Figure 6: The Atrium. The white teleportation ball and the red colored "rez" ball can be seen in the center.
Generating modules for classes or conferences is accomplished through clicking on a red sphere located in the central atrium by persons given the proper administrative authority to do so. Students and visitors can then access their desired location by clicking on a white sphere whereby they can also find out their destination through a menu and can then teleport to the appropriate location by using unique MD-5 hashed passwords, which are required for each attendant to gain access to the desired destination.

\subsection{Designing/Coding the Environment}

Due to the project being undertaken in Second Life all the code has been written in Linden Scripting Language (LSL), which is a state-event driven scripting language, with states containing descriptions on how to react to events such as receiving messages, collisions with other objects or user actions which occur while the program is within that state. As soon as a script is added to an object, and turned on, it begins to execute. LSL has a syntax similar to the $\mathrm{C}$ or Java programming languages and has an explicit state model and it models scripts as finite state machines, meaning that different classes of behaviors can be captured in separate states and that there are explicit transitions between these states. LSL has built in data types, such as vectors and quaternions, as well as a wide variety of functions for the simulation of the physical world. LSL is tightly bound to the concept of virtual-world objects in the 3D modeling sense rather than in the object-oriented sense. LSL is a strongly typed language that is compiled to byte code before runtime execution in a virtual machine on one of Linden Lab's servers. LSL's native data structure includes integers, floating point numbers, strings, keys, vectors and rotations. There are also heterogeneous lists, but no arrays. Thus, multiple lists functions must be used to adapt programs requiring array structures. Although there is no built-in database within this system scripts nonetheless continue to run even when the user is not logged in, and saved object when re-introduced into the world later, still maintain their previous state.

Currently, this project has only one variable that is shared between unlinked objects, which is the number of avatars that are colliding with any area of the building. An example to this would be that the floors and the panels of the orientation area are two different objects. Since the script in the floor will send a message to the orientation panel that a new avatar has just stepped onto the floor, the number of colliding 
avatars in the orientation panel object's script will be immediately updated.

The Reflexive Campus changes shape and size according to the number of the current users of the space. Indeed, this attribute constitutes a major component of its reflexivity. Thus the regulation of the scale of the building with respect to the number of individuals in current occupancy is achieved through a script, taking into account the number of occupants and achieving resizing of the $\mathrm{x}$ and $\mathrm{y}$ axes (NewScaleFactor

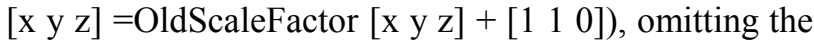
$\mathrm{z}$ axis in order to avoid the collision of floors.

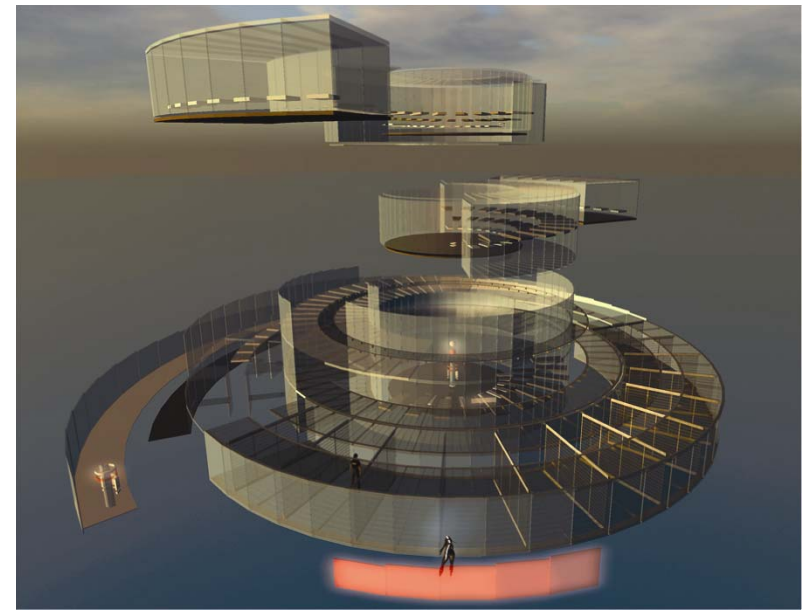

Figure 7: The Reflexive Campus, global view.

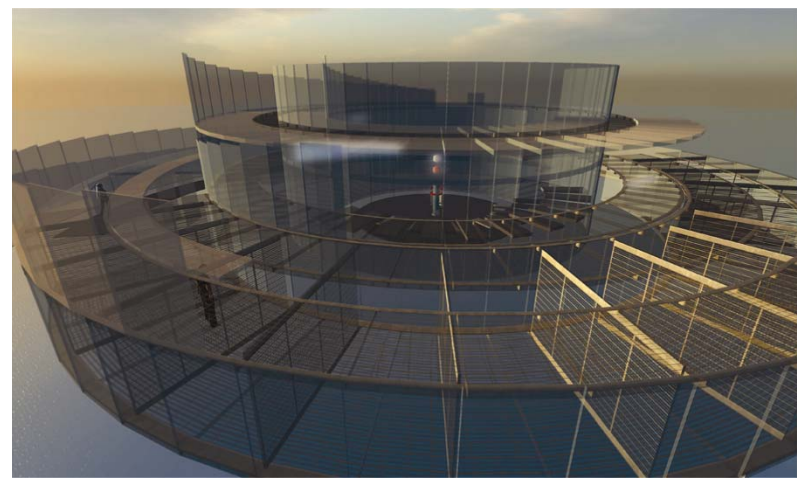

Figure 8: The wireframe exhibition panels.

The exhibition panels are located throughout the entry level hallways of the campus, forming long corridors that transfigure themselves through contact: When clicked upon these panels become nonphysical and $100 \%$ transparent for 5 seconds, allowing passage into the next section of the gallery space, thus leading the visitor along a long tunnel of progressive disclosure.

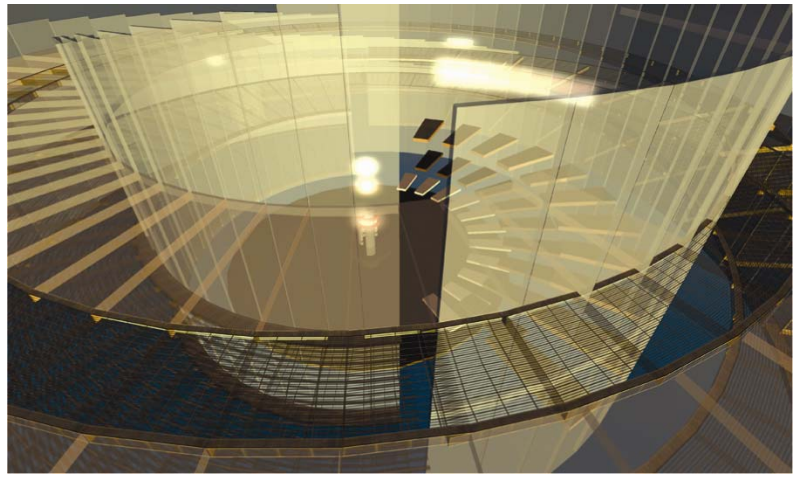

Figure 9: The glowing room creator and teleporter spheres visible at the center of the atrium.

Module creation and module access are handled by two objects, a "room creator" and a "teleporter", manifested as two small spheres, white for teleportation and red for rendering activity, in the center of the atrium. Currently the room creator can render a small room for 7 people and a larger room for 49 people. Since LSL prohibits the rendering of objects further than 10 meters away from their creators, the sphere passes an index to the newly rendered object instructing it as to the precise parameters of the location where it should be placed recursively. Beyond the location the rendered room is also endowed with a very slow moving random rotation around its z-axis. After the completed event the newly rendered room object sends back a message to the room creator which contains the key, subsequent to which the room creator object updates the room list found in its own code.

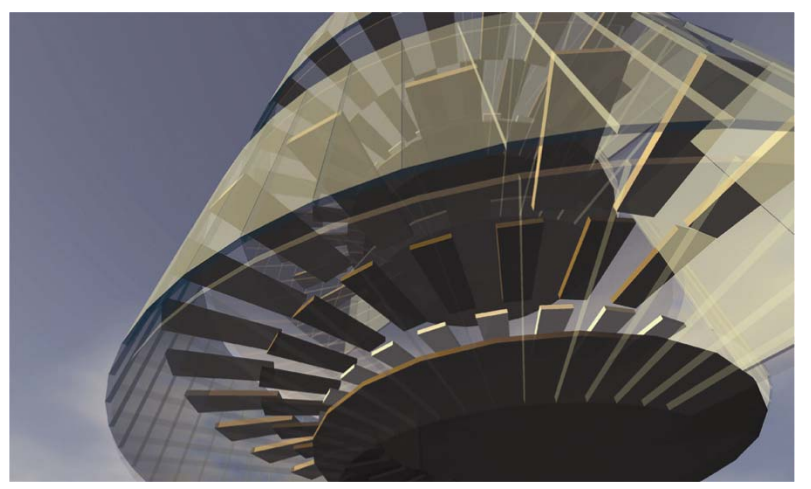

Figure 10: Detail shot of a rendered auditorium.

The modules which create the spaces of learning/research activity can be categorized as the fully circular large auditoriums for 49 users each which render themselves one above the other directly aligned to the core of the construct, thus causing the building to 
rise vertically and secondly the pie shaped outer, smaller modules designated for 7 users, which spread outwards from the center in a sunray like manner, causing the construct to expand horizontally, allowing for a virtual, structural reinterpretation of the Platonic diagram of epistemology in the allocation of types of knowledge in relation to one another.

\section{Discussion/Future Work}

At this time the Reflexive Campus only exists as a scaled down to $1 / 7$ working prototype, complete with only some of its dynamic attributes; the university that it will be dedicated to currently being in the initial phases of establishing a presence in Second Life. While the prototype will be easily implemented in terms of its overall appearance as well as the coding of its various components, such as the rendered rooms and spaces or the exhibition panels; other aspects of the project will need to get reconfigured due to some of the restrictions imposed by the Second Life building/coding platform itself where the maximum allowed linkage of objects, their size and their furthest distance from one another is concerned. Thus, the assembly of the construct will need to get reconfigured; since due to the prospective increase in dimensions, the construct will need to get segmented into components to a much larger extent than it currently is to ensure the healthy communication of the embedded scripts. It should be added here that this issue only adheres to the assembly process itself and will not affect the appearance of the building.

The team has also never yet had the opportunity of rendering the campus to its full horizontal circumference, whereby all pie shaped outer modules will need to be flung out originating from the pivot of the core structure. It is to be foreseen that the observation of this may also lead to modifications of the foreseen emergent solar structure. However beyond design and coding issues, the observation of usage will also be crucial in determining subsequent design strategies. Modules providing for larger as well as smaller gatherings than the numbers of 7 and 49 that are currently available will in all likelihood need to get added into the emergent building scheme.

The virtual campus is planned to become operational at the onset of the academic year of 2009/2010, at which point a server with a user friendly interface which will allow faculty, administrators and students to import diverse range of learning and communication materials into the system. This server system will be fully integrated with the virtual construct, providing for enhanced feedback within the virtual and the physical educational realms..

\section{References}

[01] Ayiter E., Balcisoy S., Germen M., "Propagating Collaboration: An instructional methodology for artists and engineers". Proceedings of "CC2007: Seeding Creativity: Tools, Media and Environments". ACM. USA. 2007. Pages: $45-52$.

[02] Kemp, M.,"Doing What Comes Naturally: Morphogenesis and the Limits of the Genetic Code", Art Journal, Vol. 55, 1996

[03] Maffei, A., (2006) Toyo Ito: Works Projects Writing, "Image of Architecture in the Electronic Age", Phaidon Press.

[04] Novak, M. "Liquid Architectures in Cyberspace", from "Cyberspace: First Steps" edited by Michael Benedikt 1994, p. 262

[05] Dewey, J. "Democracy and Education". Macmillan. New York. 1921. Pg: 163, 212, 245.

[06] Spiller, N., "Reflexive Architecture", Academy Press. UK, 2002

[07] Brouchoud, J., “The Arch”, http://archsl.wordpress.com/ Retrieved on: 01/12/2008. 